\title{
La Paired Comparison Technique (PCT) nella Valutazione di Impatto Ambientale di impianti idroelettrici
}

\author{
Rosanna Della Porta ${ }^{1}$, Letizia Leva ${ }^{1}$, and Fabiola Capozzoli ${ }^{1}$ \\ ${ }^{1}$ Affiliation not available
}

\begin{abstract}
Il presente lavoro mostra come la Paired Comparison Technique (PCT) si configuri come un'utile tecnica di ponderazione matriciale negli studi di impatto ambientale per l'analisi delle alternative. Sono presentati i risultati di un caso studio relativo all'implementazione della PCT in merito alla progettazione di un impianto idroelettrico, al fine di individuare l'alternativa progettuale che abbia i minori impatti potenziali sull'ambiente. In particolare, la tecnica utilizzata ha consentito di gestire criteri ed alternative in maniera obiettiva ed organica attraverso l'ordinamento di fattori in funzione del loro peso relativo. I risultati conseguiti dimostrano come la PCT sia una tecnica di facile utilizzo, ma di particolare efficacia per supportare decisioni nella Valutazione d'Impatto Ambientale.
\end{abstract}

\section{Introduzione}

L'analisi delle alternative consiste nella procedura di valutazione delle diverse scelte possibili o disponibili allo scopo di perseguire uno o più obiettivi. La letteratura offre differenti approcci per la risoluzione di problemi decisionali, tra cui le tecniche di Machine Learning (ML) e multicriteriali (MCDA) ${ }^{1}$. Nello specifico, tra le metodologie di norma impiegate negli Studi di Impatto Ambien-

tale (S.I.A.) ${ }^{2}$, quelle che consistono nell'attribuzione di pesi o magnitudo (valori d'importanza o 
incidenza) sono le più numerose e, a parità di efficacia, semplici da usare. Tra le tecniche maggiormente usate per la costruzione di una scala cardinale (elenco gerarchico e pesato) di un gruppo di fattori ambientali vi è la Paired Comparison Technique ${ }^{3}$. La PCT nota anche come metodo di comparazione a coppie è una tecnica di ponderazione o analisi delle alternative sviluppata negli anni '60 come tecnica di supporto alle decisioni. Tale metodo consente l'ordinamento di fattori o criteri in funzione della loro importanza relativa, laddove i fattori possono essere indicatori, componenti ambientali, tecnologie di mitigazione o anche opzioni progettuali ${ }^{4}$.

In ambito normativo, a livello nazionale, il D.P.C.M. 27/12/88 fornisce una linea guida circa l'organizzazione dei contenuti dello Studio di Impatto Ambientale (S.I.A.); nel quadro di riferimento progettuale rientra l'analisi delle alternative, così come specificato dall'art. 4, comma 4, dello stesso. Il D.lgs. 152/2006, così come modificato dal D.lgs. 104/2017, alla Parte II, Titolo III, art. 22, comma 3, disciplina i contenuti dello S.I.A. e in particolare rende obbligatoria l'analisi delle alternative; inoltre, l'allegato 7, all'art. 2. prevede una descrizione delle alternative prese in esame e loro comparazione con il progetto presentato. A livello Internazionale, la Direttiva 2014/52/UE, all'art. 5, paragrafo 1, stabilisce che il proponente deve includere almeno una des-

crizione delle alternative prese in esame dal committente, con indicazione delle ragioni principali alla base dell'opzione scelta, prendendo in considerazione gli effetti ambientali. In particolare, la PCT è normalizzata dalla UNI EN ISO 5495:2016.

\section{Caso studio}

Nei prossimi decenni la richiesta globale di acqua ed energia aumenterà in modo significativo ${ }^{5}$, pertanto lo sfruttamento consapevole di tali risorse strategiche rappresenta una delle più grandi sfide della società moderna ${ }^{6}$. Le fonti rinnovabili si configurano come uno strumento fondamentale per i paesi industrializzati nel raggiungere gli obiettivi di produzione di energia "pulita" ${ }^{8}$. Tra le varie tecnologie rinnovabili disponibili, gli impianti idroelettrici per la produzione di energia 
rappresentano la soluzione in grado di garantire performance migliori sia in termini di affidabilità che di riduzione delle pressioni sull'ambiente ${ }^{9}$. Il seguente caso studio vuole porre l'attenzione sull'applicazione della PCT come tecnica di ponderazione per l'analisi di tre alternative progettuali circa la realizzazione di un impianto idroelettrico ad acqua fluente al fine di individuare la configurazione avente minor impatto potenziale sull'ambiente.

Le alternative differiscono per tipologia di opera di presa e per configurazione del canale di derivazione. L'alternativa I presenta una traversa fissa con opera di presa laterale a stramazzo mentre le alternative II e III presentano entrambe una traversa di tipo a trappola con gaveta. Inoltre, l'alternativa III presenta una condotta di derivazione interrata mentre le alternative progettuali I e II sono caratterizzate da un canale di derivazione in terra (Fig. 2).

La definizione dei criteri di valutazione deriva dall' analisi dei principali impatti potenziali generati dalla realizzazione delle opere idrauliche prese in esame ${ }^{10}$. A tal fine, sono stati considerati come criteri le seguenti componenti ambientali: acqua (A), flora e vegetazione $(\mathrm{FV})$, paesaggio $(\mathrm{P})$, fauna in fase di cantiere (FC) e fauna in fase di esercizio (FE).

L'impatto sulla componente idrica (A) è associato alla tipologia di opera di presa che si intende adottare poiché ad ognuna di esse può corrispondere la necessità di progettare e realizzare differenti opere di sistemazione idraulica, le quali potrebbero rappresentare un'alterazione dello stato in cui versa il corso d'acqua. L'impatto sulla componente flora e vegetazione (FV) è connesso alle operazioni in fase di cantiere in relazione alla presenza di aree boscate o vegetazione di interesse. L' impatto sulla componente paesaggio $(\mathrm{P})$ è legato alle caratteristiche geometriche e tipologiche delle opere idrauliche. L' impatto su fauna in fase di cantiere (FC) è dovuto al disturbo che le attività di cantiere possono indurre sul naturale comportamento della fauna; in particolare, quando il cantiere permane per un tempo abbastanza lungo può succedere che le specie animali non riescano ad adattarsi al nuovo ambiente così che si generino migrazioni. Per quanto riguarda l'impatto su fauna in fase di esercizio (FE), particolare attenzione va posta alla ittiofauna, in quanto la tipologia 
di opera di presa adottata può influire sul suo libero spostamento lungo l'asta fluviale.

Definiti i criteri di valutazione, si è proceduto alla stima dei loro pesi relativi mediante la tecnica del confronto a coppie. Si è supposto di realizzare l'opera in un punto non soggetto a particolari vincoli paesaggistici e in cui non vi siano elementi di particolare pregio e soggetti a tutela; inoltre, si è deciso di dare maggior peso agli impatti permanenti su quelli temporanei. Pertanto si è considerato:

- il criterio A di maggior rilievo rispetto al criterio $\mathrm{P}$ ed FV mentre meno importante rispetto ai criteri FC e FE;

- il criterio FC meno importante rispetto al criterio FE;

- il criterio P di maggior peso rispetto al criterio FV;

- il criterio fittizio, per definizione, di minor peso rispetto a tutti gli altri criteri.

La valutazione viene effettuata mediante la costruzione di una tabella, i cui caporiga sono i diversi fattori o criteri ed in colonna si riportano i valori che scaturiscono dal loro confronto a coppie; pertanto, detto $\mathrm{N}$ il numero dei fattori considerati, il numero di coppie necessario sarà pari a N(N1)/2. Si utilizza una scala di giudizio a tre livelli, dove viene attribuito il punteggio: 1 al fattore «vincente», 0 al fattore «perdente» e 0,5 in caso di uguale importanza. Al termine del confronto sistematico tra tutte le coppie si è proceduto alla somma dei punteggi conseguiti da ciascun fattore. Infine, si è ottenuto il vettore di ponderazione dividendo ciascun punteggio per la somma totale dei punteggi (Fig. 3).

Dopodiché, si è proceduto al confronto a coppie tra le alternative progettuali per ottenere la magnitudo relativa di una alternativa rispetto alle altre in funzione di ciascun criterio. Per il criterio A, l'alternativa I risulta più importante rispetto alle alternative II e III perché in tal caso l'opera di presa a stramazzo laterale necessita di opere di mitigazione del rischio idraulico; le alternative II e III presentano medesima importanza perché sono caratterizzate dalla stessa tipologia di opera di presa (Fig. 4). 
Per quanto riguarda il criterio FE, la configurazione della opera di presa prevista nella alternativa I favorisce l'intercettazione di pesci ed il loro indirizzamento all'interno del gruppo di produzione e dunque comporta una maggiore probabilità di mortalità degli esemplari, a differenza delle alternative II e III che, presentando entrambe l'opera di presa del tipo traversa a trappola, hanno invece una limitata possibilità di intercettazione della ittiofauna (Fig. 5).

Per il criterio FC, l'alternativa III, in cui si prevede la realizzazione di una condotta di adduzione interrata, necessita di un' area di cantiere e di tempi di realizzazione maggiori rispetto alle alternative I e II e pertanto ha un'importanza maggiore (Fig. 6).

Per il criterio FV, l'alternativa III, richiedendo un'area di cantiere più grande, ha importanza maggiore, mentre l'alternativa I è più importante dell'alternativa II perché la sua opera di presa occupa un'area superiore (Fig. 7).

Infine, per quanto riguarda il criterio P, l'alternativa I ha un ingombro visuale complessivo maggiore rispetto alle altre alternative e quindi presenta un impatto maggiore (Fig. 8).

L'impatto complessivo di ciascuna alternativa è stato ottenuto come somma dei prodotti della magnitudo rispetto al criterio i-esimo per il peso relativo del criterio stesso. Dalla matrice dell'impatto complessivo risulta che l'alternativa progettuale caratterizzata dall'impatto potenziale minore è quella il cui valore è il più basso. Si deduce, pertanto, che l'alternativa II, caratterizzata da un'opera di presa con traversa a trappola e un canale di derivazione in terra, è la soluzione vincente in quanto consente di ottenere impatti potenziali minori in funzione dei criteri di selezione considerati (Fig. 9).

\section{Conclusioni}

In definitiva, il presente lavoro, attraverso il caso studio analizzato, ha mostrato l'applicazione della PCT in quanto tecnica di ponderazione e di analisi delle alternative. L'implementazione di tale 
tecnica ha permesso di valutare, in via preliminare, i possibili impatti derivanti dalla realizzazione di un impianto idroelettrico, al fine di scegliere la soluzione progettuale più sostenibile sulla quale proseguire la valutazione di impatto ambientale. La PCT si è confermata, così, un utile strumento di supporto alle decisioni.

\section{References}

1.Nesticò, A., Elia, C. \& Naddeo, V. Sustainability of urban regeneration projects: Novel selection model based on analytic network process and zero-one goal programming. Land Use Policy 99, $104831(2020)$.

2.Naddeo, V., Belgiorno, V., Zarra, T. \& Scannapieco, D. Dynamic and embedded evaluation procedure for strategic environmental assessment. Land Use Policy 31, 605-612 (2013).

3.A.Russi, F.Palmieri, D.Borchia \& E.Crescini. La Paired Comparison Technique nella VIA delle piste da sci. in Rivista di scienze ambientali vol. 1, (Researchgate, 2000).

4.V.Belgiorno, V.Naddeo \& T.Zarra. Tecniche per la valutazione di impatto ambientale. (ASTER onlus Editore, 2011).

5.Hasan, S. W., Liu, H., Naddeo, V., Puig, S. \& Yip, N. Y. Editorial: Environmental technologies for the sustainable development of the water and energy sectors. Water Science and Technology 81, iii-iv (2020).

6.Naddeo, V. \& Korshin, G. Water energy and waste: The great European deal for the environment. Science of The Total Environment 764, 142911 (2021).

7.Murena, A. et al.. Water-Energy Nexus: Evaluation of the Environmental Impact on the National and International Scenarios. in Frontiers in Water-Energy-Nexus-Nature-Based Solutions Advanced Technologies and Best Practices for Environmental Sustainability 33-35 (Springer In- 
ternational Publishing, 2019). doi:10.1007/978-3-030-13068-8.

8.Naddeo, V., Balakrishnan, M. \& Choo, K.-H. Frontiers in Water-Energy-Nexus-Nature-Based Solutions Advanced Technologies and Best Practices for Environmental Sustainability. (Springer International Publishing, 2020). doi:10.1007/978-3-030-13068-8.

9.Scannapieco, D., Naddeo, V. \& Belgiorno, V. Sustainable power plants: A support tool for the analysis of alternatives. Land Use Policy 36, 478-484 (2014).

10.F.Boano et al.. Proposte di linee guida per la valutazione degli impatti ambientali dei piccoli impianti idroelettrici. in (Reserarchgate, 2012). 


\section{Figure Captions}

Figure 1. Impianto idroelettrico ad acqua fluente (fonte: unsplash.com)

Figure 2. Alternative progettuali

Figure 3. Matrice dei confronti a coppie dei criteri

Figure 4. Matrice del confronto a coppie delle alternative rispetto al criterio A

Figure 5. Matrice del confronto a coppie delle alternative rispetto al criterio FV

Figure 6. Matrice del confronto a coppie delle alternative rispetto al criterio FC

Figure 7. Matrice del confronto a coppie delle alternative rispetto al criterio FE

Figure 8. Matrice del confronto a coppie delle alternative rispetto al criterio $\mathrm{P}$

Figure 9. Matrice dell'impatto potenziale complessivo 


\section{Figures}

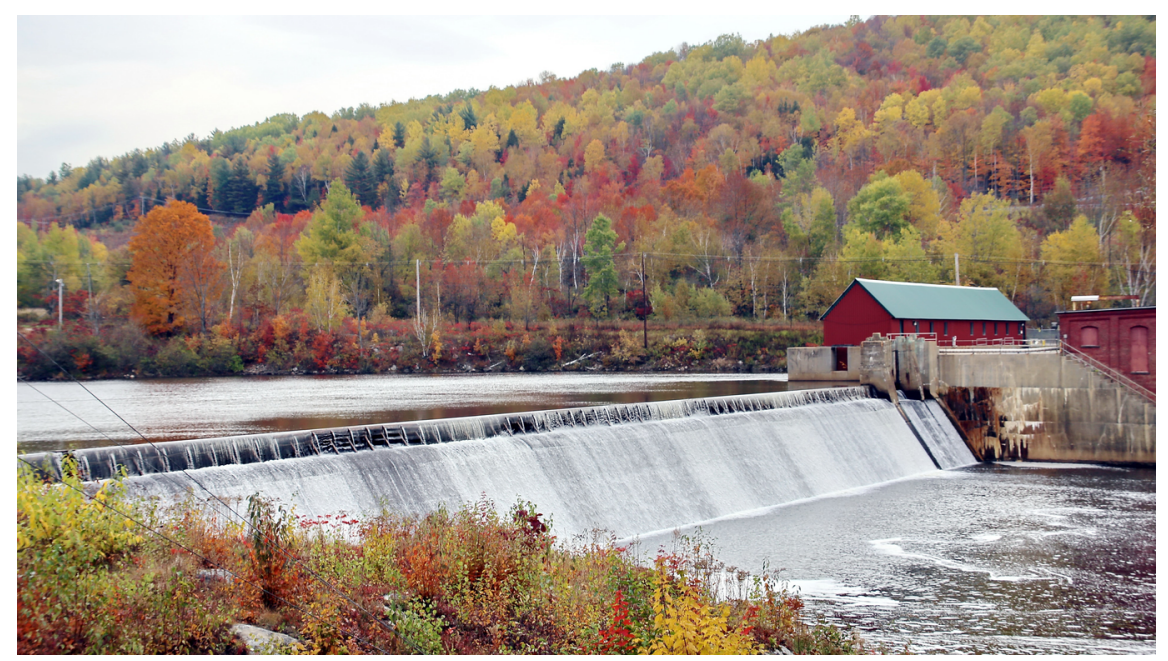

Figure 1: Impianto idroelettrico ad acqua fluente (fonte: unsplash.com)

\begin{tabular}{|c|c|c|}
\hline $\begin{array}{c}\text { Alternative } \\
\text { progettuali }\end{array}$ & Opera di presa & Canale di derivazione \\
\hline I & $\begin{array}{c}\text { Traversa fissa con opera di presa } \\
\text { laterale a stramazzo }\end{array}$ & Canale in terra \\
\hline II & Traversa a trappola con gaveta & Canale in terra \\
\hline III & Traversa a trappola con gaveta & Condotta interrata \\
\hline
\end{tabular}

Figure 2: Alternative progettuali

\begin{tabular}{|c|c|c|c|c|c|c|c|c|c|c|c|c|c|c|c|c|c|}
\hline & A-FV & A-FC & A-FE & A-P & $A-F$ & FV-FC & FV-FE & FV-P & FV-F & FC-FE & FC-P & FC-F & FE-P & FE-F & P-F & $\Sigma$ & Peso \\
\hline A & 1 & 0 & 0 & 1 & 1 & - & - & - & - & - & - & - & - & - & - & 3 & 0.20 \\
\hline FV & 0 & - & - & - & - & 0 & 0 & 0 & 1 & - & - & - & - & - & - & 1 & 0.07 \\
\hline FC & - & 1 & - & - & - & 1 & - & - & - & 0 & 1 & 1 & - & - & - & 4 & 0.27 \\
\hline FE & - & - & 1 & - & - & - & 1 & - & - & 1 & - & - & 1 & 1 & - & 5 & 0.33 \\
\hline $\mathbf{P}$ & - & - & - & 0 & - & - & - & 1 & - & - & 0 & - & 0 & - & 1 & 2 & 0.13 \\
\hline $\mathbf{F}$ & - & - & - & - & 0 & - & - & - & 0 & - & - & 0 & - & 0 & 0 & 0 & - \\
\hline & & & & & & & & & & & & & & & & 15 & 1 \\
\hline
\end{tabular}

Figure 3: Matrice dei confronti a coppie dei criteri 


\begin{tabular}{|c|cccccc|c|c|}
\hline Criterio A & I-II & I-III & I-F & II-III & II-F & III-F & $\boldsymbol{\Sigma}$ & Peso \\
\hline I & 1 & 1 & 1 & - & - & - & 3 & 0.5 \\
II & 0 & - & - & 0.5 & 1 & - & 1.5 & 0.25 \\
III & - & 0 & - & 0.5 & - & 1 & 1.5 & 0.25 \\
F & - & - & 0 & - & 0 & 0 & 0 & - \\
\hline & & & & & & & 6 & 1 \\
\hline
\end{tabular}

Figure 4: Matrice del confronto a coppie delle alternative rispetto al criterio A

\begin{tabular}{|c|cccccc|c|c|}
\hline Criterio FV & I-II & I-III & I-F & II-III & II-F & III-F & $\boldsymbol{\Sigma}$ & Peso \\
\hline I & 1 & 0 & 1 & - & - & - & 2 & 0.333 \\
II & 0 & - & - & 0 & 1 & - & 1 & 0.167 \\
III & - & 1 & - & 1 & - & 1 & 3 & 0.5 \\
F & - & - & 0 & - & 0 & 0 & 0 & - \\
\hline & & & & & & & 6 & 1 \\
\hline
\end{tabular}

Figure 5: Matrice del confronto a coppie delle alternative rispetto al criterio FV

\begin{tabular}{|c|cccccc|c|c|}
\hline Criterio FC & I-II & I-III & I-F & II-III & II-F & III-F & $\boldsymbol{\Sigma}$ & Peso \\
\hline I & 0.5 & 0 & 1 & - & - & - & 1.5 & 0.25 \\
II & 0.5 & - & - & 0 & 1 & - & 1.5 & 0.25 \\
III & - & 1 & - & 1 & - & 1 & 3 & 0.5 \\
F & - & - & 0 & - & 0 & 0 & 0 & - \\
\hline & & & & & & & 6 & 1 \\
\hline
\end{tabular}

Figure 6: Matrice del confronto a coppie delle alternative rispetto al criterio FC 


\begin{tabular}{|c|cccccc|c|c|}
\hline Criterio FE & I-II & I-III & I-F & II-III & II-F & III-F & $\boldsymbol{\Sigma}$ & Peso \\
\hline I & 1 & 1 & 1 & - & - & - & 3 & 0.5 \\
II & 0 & - & - & 0.5 & 1 & - & 1.5 & 0.25 \\
III & - & 0 & - & 0.5 & - & 1 & 1.5 & 0.25 \\
F & - & - & 0 & - & 0 & 0 & 0 & - \\
\hline & & & & & & & 6 & 1 \\
\hline
\end{tabular}

Figure 7: Matrice del confronto a coppie delle alternative rispetto al criterio FE

\begin{tabular}{|c|cccccc|c|c|}
\hline Criterio P & I-II & I-III & I-F & II-III & II-F & III-F & $\boldsymbol{\Sigma}$ & Peso \\
\hline I & 1 & 1 & 1 & - & - & - & 3 & 0.5 \\
II & 0 & - & - & 0.5 & 1 & - & 1.5 & 0.25 \\
III & - & 0 & - & 0.5 & - & 1 & 1.5 & 0.25 \\
F & - & - & 0 & - & 0 & 0 & 0 & - \\
\hline & & & & & & & 6 & 1 \\
\hline
\end{tabular}

Figure 8: Matrice del confronto a coppie delle alternative rispetto al criterio $\mathrm{P}$

\begin{tabular}{|c|ccccc|c|}
\hline & Criterio & Criterio & Criterio & Criterio & Criterio & Impatto \\
& A & FV & FC & FE & P & complessivo \\
\hline I & 0.5 & 0.33 & 0.25 & 0.5 & 0.5 & 0.42 \\
II & 0.25 & 0.17 & 0.25 & 0.25 & 0.25 & 0.24 \\
III & 0.25 & 0.50 & 0.5 & 0.25 & 0.25 & 0.33 \\
\hline Peso relativo & 0.20 & 0.07 & 0.27 & 0.33 & 0.13 & \\
\hline
\end{tabular}

Figure 9: Matrice dell'impatto potenziale complessivo 